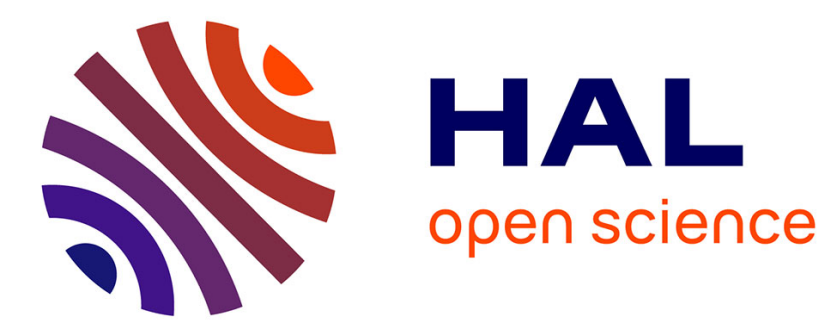

\title{
Cross-layer rate control, medium access control and routing design in cooperative VANET
}

Liang Zhou, Baoyu Zheng, Benoit Geller, Anne Wei, Shan Xu, Yajun Li

\section{To cite this version:}

Liang Zhou, Baoyu Zheng, Benoit Geller, Anne Wei, Shan Xu, et al.. Cross-layer rate control, medium access control and routing design in cooperative VANET. Computer Communications, 2008, 10.1016/j.comcom.2007.12.006 . hal-01248385

HAL Id: hal-01248385

https://hal-ensta-paris.archives-ouvertes.fr/hal-01248385

Submitted on 25 Dec 2015

HAL is a multi-disciplinary open access archive for the deposit and dissemination of scientific research documents, whether they are published or not. The documents may come from teaching and research institutions in France or abroad, or from public or private research centers.
L'archive ouverte pluridisciplinaire HAL, est destinée au dépôt et à la diffusion de documents scientifiques de niveau recherche, publiés ou non, émanant des établissements d'enseignement et de recherche français ou étrangers, des laboratoires publics ou privés. 


\title{
Cross Layer Rate Control, Medium Access Control and Routing Design in Cooperative VANET
}

Liang Zhou ${ }^{1,2}$, Baoyu Zheng ${ }^{2}$, Benoit Geller ${ }^{3}$, Anne $\mathrm{Wei}^{3}$, Shan $\mathrm{Xu}^{2}$, Yajun $\mathrm{Li}^{1}$

1. Electrical Engineering Department, Shanghai Jiao Tong University, 200240, China

2. Institute of Signal Processing and Transmission, Nanjing University of Posts and Telecommunications, 210003, China

3. Laboratory of SATIE, Ecole Normale Supérieure de Cachan, 94235, France

\begin{abstract}
In this paper, we address the rate control, the Medium Access Control (MAC) and the routing problem for cooperative Vehicular Ad-Hoc Network (VANET) in the framework of cross-layer design. At first, we introduce the cooperative communication conception to VANET, and propose an opportunistic cooperation strategy to improve the system performance. And then, we develop a cross-layer solution which consists of the link capacity detection with adjusting persistence probability at the MAC Layer, the flow rate control with the maximal utility at the Transport Layer and the routing design at the Network Layer. This proposal is designed in distributed manner in order to support a simple and efficient implementation for VANET. Furthermore, some practical issues, such as fairness and network cost, are presented for implementing the proposed solution and improving the system performance. Simulation results show that the proposed opportunistic cooperation strategy combined with joint control algorithm achieves the desired performance over VANET.
\end{abstract}

Keywords: vehicular ad-hoc network, cooperative, medium access control, rate control, routing 


\section{Introduction}

Vehicular Ad-Hoc Network (VANET) is an emerging new type of wireless networks in which vehicles constitute the mobile nodes in the network. This kind of networks supports vehicle-to-vehicle and vehicle-to-gateway communications by providing a self-organized and multi-hop network. The main technical challenge for communication in VANET is the strong mobility of the nodes, high dynamic change of the topology, high variability in node density, and very short duration of the communication.

Let's first consider classic VANET with short-range communication. There are wireless gateways at regular intervals providing access to the rest of the Internet using infrastructure support. Given an average speed of 50 miles per hour and a gateway radio range of 500 meters, every vehicle with the transmission window connected to a fixed Internet access point has one minute at the most. Considering contention from other vehicles, there may not be enough bandwidth to allow each vehicle to access the Internet in the short time. From the above example, we can find that conventional client-server approach in the face of intermittent connectivity is not appropriate for VANET. Therefore, limited access to the Internet contrasts with the even increasing dependence on Internet service, which motivates a compelling application of cooperative network in the VANET. In this cooperative network, every node (vehicle) acts as a partner for other nodes in a multi-hop wireless scenario. Thus, cooperation at the network level can be resonated at the application level [1]. 
In fact, the idea of cooperative networking is first proposed to handle "flash crowds" on the Internet, where end-hosts cooperate in order to improve the overall network performance. The gateway in our architecture would experience flash crowd arrival patterns. As we shall see, the strong mobility of the nodes in VANET coupled with the intermittent connectivity to the Internet provides an incentive for individual nodes to cooperate while accessing the Internet to achieve some level of seamless connectivity.

In recent years, some cooperative strategies for content delivery and sharing in VANET have been proposed. Among these, [2] proposes a "communication efficient" swarming protocol which uses a gossip mechanism that leverages the inherent broadcast nature of the wireless medium, and a piece-selection strategy that takes proximity into account in decisions to exchange pieces. However, there is no specific solution describing how to solve the data flow movement problem over this multiple hop network. In fact, since cooperative VANET is a classic multi-source-multi-path system, one of most challenge issues is the flow rate control: the Internet is based on an end-to-end paradigm, where the transport protocol (e.g. TCP) instances at the endpoints to detect overload conditions at intermediate nodes. When congestion occurs, the source reduces flow rate [3]. However, in cooperative VANET the topology changes within seconds and the congested nodes might not be adjusted at all when the sources react to the congestion.

In this paper, we jointly formulate the rate control, medium access control and routing problem for cooperative VANET in the framework of the utility function 
optimization. The fundamental purpose is to decompose the utility function into a flow control problem which determines the total source rate, and a division problem which describes how to split the total rate among a set of least congested paths according to the link persistence probability. The main contributions of this research are showed as follows: Firstly, we introduce the cooperative communication conception to VANET, and propose an opportunistic cooperation strategy to improve the system performance. Secondly, we develop a cross-layer solution which consists of the link capacity detection with adjusting persistence probability at the MAC Layer, the flow rate control with the maximal utility function value at the Transport Layer and the routing at the Network Layer. Generally speaking, the realization of joint rate control, MAC control and routing for this specific cooperative VANET system is the highlight of this paper. Finally, some practical issues for implementing the proposed scheme and improving the VANET performance are presented.

The remainder of this paper is organized as follows. Section 2 introduces the opportunistic cooperation strategy for VANET. Based on the problem analysis, a joint optimal rate control, medium access control and routing algorithm is proposed in Section 3. In Section 4, some practical implementation issues for VANET are presented. Simulation result and discussion are given in Section 5, followed by related works in Section 6 and concluding remarks in Section 7.

\section{Opportunistic Cooperation Strategy for VANET}

The VANET system consists of a set of nodes communicating through bidirectional 
wireless links between each other with the $5.9 \mathrm{GHz}$ Dedicated Short-Range Communication (DSRC) [4]. The DSRC link is compatible with IEEE 802.11a R/A (Roadside Applications) by the Standard Committee E17.51.

The basic opportunistic cooperation strategy in this research is described as follows: when a vehicle arrives in the range of the gateway $\left(R_{g}\right)$, it requests the gateway for the particular data. If the gateway has the data in its cache, it starts downloading from the Gateway while in $R_{g}$ (Figure 1(a)). When the vehicle gets out of the range of $R_{g}$, it starts to find his partner by gossip its neighbors about content availability. If the wanted data exists in its neighbor and the neighbor can provide a satisfactory service, the neighbor transmits the data in the range of the vehicle $\left(R_{v}\right)$ (Figure 1(b)). If his neighbor has the wanted data, but it can not provide satisfactory service, the neighbor seeks to relay for help (Figure 1(c)). Otherwise, the neighbor gossip to its neighbors and become the potential relay for this vehicle (figure 1(d)). In the context of Figure 1(b) and 1(c), it is important for a vehicle to decide whether employing a relay or not. To resolve this problem, we focus on designing an Opportunistic Cooperation MAC (OC-MAC) protocol which is specially adapted to VANET.

\subsection{Relay Employment}

Since the throughput is one of the most important indexes for VANET, here, we choose it as the criterion of relay employment. That is, relay nodes will be used only when they can improve the throughput of the system. The throughput of the system $T$ is defined as: 


$$
T=\frac{P_{f} P_{\text {size }}}{t}
$$

$t$ represents the transmission time of a frame (including the control frame and the data frame), $P_{\text {size }}$ is the size of each packet and $P_{f}$ represents the possibility of successful transmission of packet in one transmission. $P_{f}$ can be calculated by the BER (Bit Error Rate) and frame length as introduced in [5]. Without considering the transmission time of SIFS (Short Inter Frame Space) slots in the IEEE 802.11 network, the transmission time $t$ can be defined as:

$$
t=t_{c}+t_{d}
$$

$t_{c}$ denotes the transmission time of control frames, where $t_{c}=t_{r t s}+t_{c t s}\left(t_{r t s}\right.$ is the time of RTS (Request To Send); $t_{c t s}$ is the time of CTS (Clear To Send)). $t_{d}$ represents the transmission time of data frame and ACK (acknowledgment) frame, where $t_{d}=t_{d a t a}+t_{a c k}$. In addition, $t_{s}$ (see equation (3)) denotes the transmission time when direct transmission is successful, and $t_{r}$ (see equation (4)) defines the transmission time when direct transmission is failed and relay is used,

$$
\begin{aligned}
& t_{s}=t_{c}^{\prime}+t_{d} \\
& t_{r}=t_{c}^{\prime}+t_{d}^{\prime}
\end{aligned}
$$

according to IEEE 802.11 standard, $t_{c}^{\prime}=t_{r t s}+t_{c t s}+t_{r c}\left(t_{r c}\right.$ is the time of RC (Relay Confirmation)), $t_{d}^{\prime}=2 \times t_{\text {data }}+t_{a c k}$. So the throughput of relay strategy $T_{r}$ can be expressed as:

$$
T_{r}=\frac{P_{f} P_{\text {size }}}{t_{s}}+\frac{\left(1-P_{f}\right) P_{r} P_{\text {size }}}{t_{r}}
$$

$P_{r}$ is the possibility of successful transmission through relay node. Relay is used only when $T_{r}>T$, and this decision is made by the intermediate destination node. 
In our scheme, relay selection is based on instantaneous channel conditions in slow fading environment, without requiring the knowledge of the topology. Meanwhile, the overhead involved is minimal since the technique is based on signal strength measurement of RTS and CTS packets. The transmission of these packets allows for the estimation of the instantaneous wireless channel information $h_{i, j}$ from node $i$ to node $j$, therefore, each node can establish a one hop routing table and choose appropriate nodes for data relaying.

\subsection{The OC-MAC Protocol}

Once a node $S$ has the data to transmit to a destination node $D$, it will first send the data to $D$ if the connection is not failed. As the topology changes so fast and the relay nodes are often used in VANET. Therefore, $S$ also checks its relay table to choose a relay with best channel condition if the table is not empty.

In the case of the relay node $R$ is used, $S$ senses the channel first to see if it is idle for a DIFS (Distributed Inter-Frame Space) time according to IEEE 802.11 standard. When $S$ has completed the required back-off procedure, a RTS frame will be sent, including the MAC address of the relay node, and this frame also reserves the channel for NAV (Network Allocation Vector) duration. The potential relay node will check its own state to see if it can relay the information for the sender, if so, a RC frame will be sent to the destination node in a SIFS time slot after received the RTS frame. Otherwise, it will just stay idle.

After hearing the $\mathrm{RC}$ frame, the destination node $D$ will use the channel information $h_{S, D}$ (from source to destination) and $h_{R, D}$ (from relay to destination) to 
calculate the possibilities $P_{f}$ and $P_{r}$ [5]. With the transmission time of control frame and data frame, $T$ and $T_{r}$ can be finally computed and compared to decide whether to use a relay node or not. If a relay node is used, a confirm signature will be specified in the CTS frame, which informs the sender the use of relay. Otherwise, a CTS frame will also be sent out but without a confirmation. In this situation, the sender will delete this relay node from the table after the reception of CTS frame.

If a relay node $R$ correctly receives the data frame, it senses the channel to see if there is an ACK frame. If so, that means the destination node $D$ has received the data frame successfully, and the relay node $R$ does not have to send the data frame again. If an ACK frame is not heard in a two SIFS time, the relay node $R$ will send the data frame to the destination node $D$. If the destination node $D$ successfully receives this data frame, it will send out an ACK frame to indicate a successful transmission. Figure 2 depicts this procedure.

\subsection{NAV and TIMEOUT Setting}

In the IEEE 802.11 standard, the original RTS and CTS frame have a duration field to indicate how long this transmission will use the channel and to keep other nodes in the BSS (Basic Service Set) silent during the transmission. The NAV at each station should be set by the value of duration field of the received frame. The node will also set a timeout clock to wait for the reply. For example, after the sender sends a RTS frame, it will set a timeout clock to wait for a CTS frame. If the CTS frame does not arrive in the regular time, the sender will retransmit another RTS frame. 
In our OC-MAC protocol, the duration field in RTS frame Duration $_{R T S}$ includes the time required to transmit the data frame through direct transmission, three SIFS intervals, one CTS frame and one ACK frame (see equation (6)). The timeout of RTS frame Timeout $_{\text {RTS }}$ includes the time of two SIFS intervals, one RC frame and one CTS frame (see equation (7)).

$$
\begin{gathered}
\text { Duration }_{R T S}=3 \times T_{S I F S}+T_{C T S}+T_{D A T A}+T_{A C K} \\
\text { Timeout }_{R T S}=2 \times T_{S I F S}+T_{R C}+T_{C T S}
\end{gathered}
$$

This duration field guarantees that a packet can be directly sent even if the relay node can not be used. The timeout clock guarantees that the sender will wait enough time for the RC and CTS frames. The duration field in RC frame Duration $_{R C}$ will be set according to the following equation:

$$
\text { Duration }_{R C}=4 \times T_{S I F S}+T_{C T S}+2 \times T_{D A T A}+T_{A C K}
$$

When setting the duration field in the CTS frame, we have to check out if the RC frame has been received. If not, we can set the value by subtracting the time for a SIFS interval and a CTS frame. If RC frame is present, the duration in the CTS Duration $_{C T S}$ and timeout Timeout ${ }_{C T S}$ should be set as below:

$$
\begin{gathered}
\text { Duration }_{C T S}=3 \times T_{\text {SIFS }}+2 \times T_{D A T A}+T_{A C K} \\
\text { Timeout }_{C T S}=3 \times T_{\text {SIFS }}+2 \times T_{D A T A}
\end{gathered}
$$

The NAV mechanism for OC-MAC is shown in Figure 3. The timeout set guarantees the destination node can receive the data frame transmitted by the relay node. For those nodes that can hear both RTS and CTS frames, they have to set their NAV according to the duration field. Once the CTS or ACK packet is captured, they might reset the NAV according to this new message. Since the relay node may be 
failed or direct transmission might be successful, the duration fields in the new packets may reserve less time.

If the data packet is successfully transmitted, every station proceeds to start a new cycle, shown in Figure 4. If the deliver of data packet is failed, the sender will start an exponential back-off to retransmit its data.

\subsection{Comparison with Other Ad Hoc Routing Algorithms}

Here we analyze our proposed OC-MAC protocol by comparing it with some ad hoc routings, such as the AODV (Ad hoc On-Demand Distance Vector) and OLSR (Optimized Link State Routing) protocols. AODV is a reactive routing protocol, in which it enables to build routes between nodes only as desired by source nodes. It maintains these routes as long as they are needed by the sources; In terms of OLSR, as it is a proactive routing protocol, each node exchanges the topology information regularly and the routes are always immediately available when needed. However, the topological changes may cause the flooding of the topological information in the network even if the "relay" technique is used in OLSR.

In fact, our proposed OC-MAC protocol is designed to cater to the characteristics of VANET. Firstly, AODV is not totally suitable any more as the topology of the VANET could be changed frequently. In OC-MAC protocol, we employ hop-by-hop strategy in which each node just knows its next hop. Although it may be could not guarantee the whole routing is optimal, it enables to provide an existed routing from source to destination under the condition of fast change environment, such as VANET; Secondly, comparing to OLSR the ultimate destination node decides the routing, 
OC-MAC protocol chooses the routing locally in which the intermediate destination node decides whether a relay is employed or not. Therefore, the amount of topological information can be reduced, which is important for VANET.

\section{Joint Optimal Control Algorithm}

In the previous section, we introduce the opportunistic cooperation strategy for the VANET. In fact, very node in this cooperative network is possible to become the source, relay or destination, and this is a classic complex multi-source-multi-path system, therefore, it is much more difficult to control the network compared to the traditional non-cooperative system. In this section, we present a Joint Optimal Control (JOC) algorithm to jointly optimize rate control, medium access control and routing for the aforementioned cooperative VANET system based on the cross-layer design.

\subsection{System Analysis}

Consider this VANET whose links are denoted by $L=\{1,2, \ldots l\}$. Let $c_{l}$ be the capacity of link $l \in L$ and $c=\left[c_{1}, c_{2}, \ldots c_{l}\right]^{T}$. Let $S=\{1,2, \ldots s\}$ be the set of sources. Each source $s \in S$ has $n_{s}$ available paths or routers from the source to the destination. Let the $L \times 1$ vector $R_{s, i}$ denotes the set of links used by source $s \in S$ on its path $i \in\left\{1,2, \ldots n_{s}\right\}$, whose $l$ th element equals to 1 if path contains link $l$ and 0 otherwise, and $N_{s, i}$ denotes the number of links on the path $i$. The set of all the available paths of $s$ is defined by

$$
R_{s}=\left[R_{s, 1}, R_{s, 2}, \ldots, R_{s, n_{s}}\right]
$$


And the total paths in the network are defined by a routing matrix $R$,

$$
R=\left[R_{1}, R_{2}, \ldots, R_{s}\right]
$$

For each source $s \in S$, let $x_{s, i}$ be the rate of source $s$ on path $R_{s, i}$, and $x_{s}=\sum_{i=1}^{n_{s}} x_{s, i}$ be the total source rate. Let $m_{s} \geq 0$ and $M_{s} \leq \infty$ be minimum and maximum rate, respectively, i.e., $m_{s} \leq x_{s} \leq M_{s}$. When each source $s$ transmits at a total rate of $x_{s}$, it attains a utility $U_{s}\left(x_{s}\right)$. We assume that $U_{s}: \mathfrak{R}_{+} \rightarrow \mathfrak{R}$ is continuous, increasing and strictly concave. Let

$$
x=\left[x_{1,1}, \ldots, x_{1, n_{1}}, x_{2,1}, \ldots, x_{2, n_{2}}, \ldots, x_{n, 1}, \ldots, x_{n, n_{s}}\right]^{T} \in \mathfrak{R}_{+}^{R}
$$

be the vector of all path rates of all sources. Our objective is to choose rates $x$ so as to maximize the total utility $\sum_{s \in S} U_{s}\left(x_{s}\right)$ :

$$
\begin{gathered}
\max _{x_{s} \geq 0} \sum_{s \in S} U_{s}\left(x_{s}\right) \\
\text { subject to } \quad x_{s}=\sum_{i=1}^{n_{s}} x_{s, i}, \\
R x \leq c \\
x_{s, i} \geq 0, m_{s} \leq x_{s} \leq M_{s}
\end{gathered}
$$

However, in the cooperative system, each node or each link has a contention resolution protocol based on the transmission persistence probability. Let $L_{\text {out }}(s)$ denotes the set of outgoing links from $s \in S, N_{t o}^{I}(l)$ as the set of nodes whose transmissions cause interference to the receiver of link $l$, excluding the transmitter node of link, and $L_{\text {from }}^{I}(s)$ as the set of links whose transmissions get interfered from the transmission of node $s$, excluding the outgoing links from node $s$. Therefore, if the transmitter of link $l$ and a node in $N_{t o}^{I}(l)$ transmit data simultaneously, the transmission of link $l$ fails. If node $s$ and the transmitter of a 
link $l$ in $L_{\text {from }}^{I}(s)$ transmit data simultaneously, the transmission of link $l$ also fails. Assuming each $s$ transmits data with a probability $p_{s}^{\prime}$, when it determines to transit data, it chooses one of its outgoing paths with a probability $q_{l}, l \in L_{\text {out }}(s)$. Consequently, link $l \in L_{\text {out }}(s)$ transmits data with a probability $p_{l}=p_{s}^{\prime} q_{l}$, which is called persistence probability of link $l$. Therefore, the data rate on path $i, x_{s, i}$, is obtained as minimum rate of link in the path:

$$
x_{s, i}\left(P_{s}\right)=\min _{l \in\left[1, N_{s, i}\right]} c_{l} p_{l} \prod_{k \in N_{\text {to }}^{I}(l)}\left(1-\sum_{m \in L_{\text {out }}(k)} p_{m}\right)
$$

where $P_{s}=\left[p_{1}, \ldots, p_{N_{s, i}}\right], P=\left[P_{1}, P_{2}, \ldots, P_{s}\right]$.

With the above analysis, we can specify the cooperative wireless networks utility maximization with "contention" link:

$$
\begin{gathered}
\max _{x_{s} \geq 0} \sum_{s \in S} U_{s}\left(x_{s}\right) \\
\text { subject to } \quad x_{s}=\sum_{i=1}^{n_{s}} x_{s, i}\left(P_{s}\right), \\
R x \leq c \\
x_{s, i} \geq 0, m_{s} \leq x_{s} \leq M_{s}, 0 \leq P \leq 1
\end{gathered}
$$

\subsection{JOC Algorithm}

In the case of multi-path flow control, many literatures have been proposed by introducing the variable of path price to maximum the total utility function of the system [6-8]. In [7], the author solves the optimal congestion control problem by taking the path price into consideration, and the optimal source rate $x_{s}^{*}$ is given by

$$
x_{s}^{*}=\sum_{R_{s, i}^{*} \in R_{s}^{*}} x_{s}^{*}=\left[U_{s}^{\prime-1}\left(p_{s}^{r^{*}}\right)\right]_{m_{s}}^{M_{s}} \text { and } x_{s, i}=0, \text { if } p_{s, i}^{r}>p_{s}^{r^{*}}
$$

where $[z]_{m_{s}}^{M_{s}}=\max \left(m_{s}, \min \left(M_{s}, z\right)\right), p_{s}^{r^{*}}$ is the minimum path price among $R_{s}$, path 
$R_{s, i}^{*}$ has the minimum path price $p_{s, i}^{r^{*}}=p_{s}^{r^{*}}$, and $R_{s}^{*}$ defines the set of all minimum price path $R_{s, i}^{*}$ of source $s$. The above equation suggests a way to adapt that the total source to congestion, but it does not specify the total rate should be split among the available paths. A naïve approach is to simply split it evenly along paths that have the least current price. This algorithm, however, does not converge, e.g. when multiple paths have different path prices. Here, we can view the persistence probability as a special path price, and the distributed optimal $x_{s}$ at each source can be adjusted according to the persistence probability of each link. Similarly, the persistence probability is adjusted according to current $x_{s}$. Intuitively, the expected result would allocate the "right" amount persistence probability to the "right" links to control the flow rate, which may then induce an increase in end-to-end throughput.

JOC Algorithm: We assume that time is slotted, and the links are fixed within a time slot but independently change between different slots. During each time slot $t$, the following three updates are carried out simultaneously until convergence.

1) MAC: Each link $l$ receives flow rates $x_{s, i}(t)$ for all paths $R_{s, i}$ that contain link $l$, and computes a new persistence probability

$$
p_{l}(t+1)=\left[p_{l}(t)+\kappa \frac{c_{l}-\sum_{s} R_{s, i} x}{p_{l}(t)}-\kappa \frac{\sum_{k \in L_{\text {from }}^{I}\left(t_{l}\right)} \varepsilon_{k}}{1-\sum_{m \in L_{\text {out }}\left(t_{l}\right)} p_{m}}\right]_{0}^{1}
$$

$t_{l}$ is the transmitter node whose path contains link $l, \kappa>0$, and

$$
\varepsilon_{l}=\left\{\begin{array}{l}
0, \quad \text { if } \sum_{i} x_{s, i} \leq c_{l} p_{l} \prod_{k \in N_{\text {lo }}^{I}(l)}\left(1-\sum_{\begin{array}{c}
m \in L_{\text {out }}(k) \\
\text { otherwise }
\end{array}} p_{m}\right) \\
1,
\end{array} .\right.
$$

Informing new probability $p_{l}(t+1)$ to the other sources whose path $R_{s, i}$ contains $\operatorname{link} l$; and each source receives $p_{s, i}^{r}(t)=P_{s}^{T}(t) R_{s, i}$ from the network for all its paths $R_{s, i}$, 
$i=1,2, \ldots, n_{s}$ and decides the maximum persistence probability

$$
p_{s}^{r^{*}}(t)=\max _{i=1,2, \ldots, n_{s}} p_{s, i}^{r}(t)
$$

2) Rate Control: Updates the source rate $x_{s}(t+1)$ :

$$
x_{s}(t+1)=\left[U_{s}^{\prime}\left(p_{s}^{r^{*}}(t)\right)\right]_{m_{s}}^{M_{s}}
$$

To these paths which do not have the maximum persistence probability, path rate

$$
\begin{aligned}
& x_{s, i}(t+1) \text { on path } R_{s, i} \text { is: } \\
& \qquad x_{s, i}(t+1)=\left[x_{s, i}(t)-\gamma\left(p_{s}^{r^{*}}(t)-p_{s, i}^{r}(t)\right)\right]^{+}, \quad \gamma>0
\end{aligned}
$$

To the any path $R_{s, j}$ that has the maximum persistence probability, we set its rate as:

$$
x_{s, j}(t+1)=\left[x_{s}(t+1)-\sum_{\substack{i \in\left[1, n_{s}\right] \\ i \neq j}} x_{s, i}(t+1)\right]^{+}
$$

and then informs all the new flow rate $x_{s, i}(t+1)$ to links $l$ contained in path $R_{s, i}$

3) Routing: Over the chosen link, sending an amount of bits for destination according to the rate determined by the rate control update.

Note that, the persistence probability of the link depends on two aspects: one is the current flow rate relative to link capacity, while the other one is the current link affected by other vehicles. In the case of path rate, the rates on all paths that have less than the maximum persistence probability are reduced by an amount proportional to the absent probability, and the rate on the maximum probability path is increased, so that the new rates on all paths sum up to the new total source rate determined in the flow control decision.

\subsection{Performance Evaluation}

We first associate Lagrange multiplier for each of the constraints, and use the KKT 
optimality conditions for optimization [9-10], solving this problem is equivalent to satisfying the complementary slackness condition and finding the stationary points of the Lagrange.

$$
\begin{aligned}
L_{s y s} & =L(x, P, \bar{\lambda}, \underline{\lambda}) \\
& =\sum_{s \in S} U_{s}\left(x_{s}\right)-\bar{\lambda}_{s}\left(x_{s}-\sum_{i=1}^{n_{s}} x_{s, i}\left(P_{s}\right)\right)-\underline{\lambda}_{l}^{T}(R x-c) \\
& =\left(\sum_{s \in S} U_{s}\left(x_{s}\right)-\bar{\lambda}_{s} x_{s}\right)+\bar{\lambda}_{s} \sum_{i=1}^{n_{s}} x_{s, i}\left(P_{s}\right)-\underline{\lambda}_{l}^{T}(R x-c)
\end{aligned}
$$

where $\bar{\lambda}=\left[\bar{\lambda}_{1}, \bar{\lambda}_{2}, \ldots, \bar{\lambda}_{s}\right], \quad \underline{\lambda}=\left[\underline{\lambda}_{1}, \underline{\lambda}_{2}, \ldots, \underline{\lambda}_{l}\right]^{T}$. By linearity of the differentiation operator, this can be decomposed into two separate maximization problems

$$
\begin{gathered}
\max _{x \succ=0} L_{x}(x, \bar{\lambda})=\sum_{s \in S} U_{s}\left(x_{s}\right)-\bar{\lambda}_{s} x_{s} \\
\max _{P \succ=0} L_{P}(\bar{\lambda}, \underline{\lambda}, P)=\bar{\lambda}_{s} \sum_{i=1}^{n_{s}} x_{s, i}(P)-\underline{\lambda}_{l}^{T}(R x-c) \\
=\bar{\lambda}_{s} \sum_{i=1}^{n_{s}}\left(\min _{l \in\left[1, N_{s, i}\right]} c_{l} p_{l} \prod_{k \in N_{l o}^{I}(l)}\left(1-\sum_{m \in L_{o u t}(k)} p_{m}\right)\right)-\underline{\lambda}_{l}^{T} p_{l}\left(x_{l}-c_{l}\right)
\end{gathered}
$$

The first maximization is already implicitly solved by the rate control mechanism for different $U_{s}$ in [11], while the second maximization still need to be solved which aims at allocating exactly the right persistence probability of each link to achieve the optimal rate. Here, we focus on proving the convergence of the persistence probability problem.

Because $\max L_{P}(P)$ is a typical non-concave/convex problem, it is difficult to take the derivative of $L_{P}(P)$ with respect to $p_{l}$ directly. To get around the difficulty, we take logarithm operator on $L_{P}(P)$ to get $L_{P}(\tilde{P})$, that is $\left.L_{P}(\tilde{P})=\min _{l \in\left[1, N_{s, i}\right]} \sum_{i=1}^{n_{s}} \log \left(c_{l} p_{l} \prod_{k \in N_{\text {lo }}^{l}(l)}\left(1-\sum_{m \in L_{\text {out }}(k)} p_{m}\right)\right)+\log p_{l}\left(c_{l}-x_{l}\right)\right], \quad$ where $\tilde{p}_{l}=\log p_{l}$. In the appendix, we will show that the partial Lagrange is a strictly concave function 
of a logarithmically transformed probability vector. So we can decompose that

$$
\begin{aligned}
L_{P}(\tilde{P}) & =\min _{l \in\left[1, N_{s, i}\right]} \sum_{i=1}^{n_{s}} \log \left(c_{l} p_{l} \prod_{k \in N_{\text {lo }}^{I}(l)}\left(1-\sum_{m \in L_{\text {out }}(k)} p_{m}\right)\right)+\log p_{l}\left(c_{l}-x_{l}\right) \\
& =\sum_{i=1}^{n_{s}} \log \left[c_{l} p_{l} \prod_{k \in N_{\text {lo }}^{I}(l)}\left(1-\sum_{m \in L_{\text {out }}(k)} p_{m}\right)-\sum_{n \in L_{\text {from }}^{I}\left(t_{l}\right)} c_{n} p_{n} \prod_{k \in N_{\text {to }}^{I}(n), k \neq t_{l}}\left(1-\sum_{m \in L_{\text {out }}(k)} p_{m}\right)\right] \\
& +\log p_{l}\left(c_{l}-\sum_{s} R_{s, i} x\right)
\end{aligned}
$$

Assuming

$$
\varepsilon_{l}=\left\{\begin{array}{c}
0, \quad \text { if } \sum_{i} x_{s, i} \leq c_{l} p_{l} \prod_{k \in N_{l o}^{I}(l)}\left(1-\sum_{m \in L_{\text {out }}(k)} p_{m}\right) \\
1, \text { otherwise }
\end{array},\right.
$$

Taking the derivative of $L_{P}(\tilde{P})$ with respect to $\tilde{p}_{l}$, we have

$$
\begin{aligned}
\nabla_{l} L_{P}(\tilde{P}) & =\left(c_{l}-\sum_{s} R_{s, i} x\right)-p_{l} \frac{\prod_{k \in N_{\text {lo }}^{I}(l)}\left(1-\sum_{m \in L_{\text {out }}(k)} p_{m}\right) \varepsilon_{k}}{1-\sum_{m \in L_{\text {out }}\left(t_{l}\right)} p_{m}} \\
& =\left(c_{l}-\sum_{s} R_{s, i} x\right)-p_{l} \frac{\sum_{k \in L_{\text {from }}^{I}\left(t_{l}\right)} \varepsilon_{k}}{1-\sum_{m \in L_{\text {out }}\left(t_{l}\right)} p_{m}}
\end{aligned}
$$

Coming back to the $P$ solution space instead of $\tilde{P}$, it is easy to verify that the derivate of $L_{P}(P)$ with respect to $p_{l}$ is

$$
\nabla_{l} L_{P}(P)=\frac{c_{l}-\sum_{s} R_{s, i} x}{p_{l}}-\frac{\sum_{k \in L_{\text {from }}^{I}\left(t_{l}\right)} \varepsilon_{k}}{1-\sum_{m \in L_{\text {out }}\left(t_{l}\right)} p_{m}}
$$

Therefore, the logarithmic change of variables simply scales each entry of the gradient by $p_{l}: \nabla_{l} L_{P}(P)=(1) /\left(p_{l}\right) \nabla_{l} L_{P}(\tilde{P})$. We now use the gradient method [12], with a constant step size $\kappa$, to maximize $L_{P}(P)$

$$
\begin{aligned}
p_{l}(t+1) & =p_{l}(t)+\kappa \nabla_{l} L_{P}(P) \\
& =\left[p_{l}(t)+\kappa \frac{c_{l}-\sum_{s} R_{s, i} x}{p_{l}(t)}-\kappa \frac{\sum_{k \in L_{\text {from }}^{I}\left(t_{l}\right)} \varepsilon_{k}}{1-\sum_{m \in L_{\text {out }}\left(t_{l}\right)} p_{m}}\right]_{0}^{1}
\end{aligned}
$$

This is the exact MAC update in the JOC algorithm. Substituting the given equation 
into $x_{s}(t+1)=\left[U_{s}^{\prime}\left(p_{s}^{r^{*}}(t)\right)\right]_{m_{s}}^{M_{s}}$ to compute the total rate of source $s$ at $t+1$, the following is the same as the proof in [6]. Since $x_{s, i}$ can be turned into a concave function in $P$, each constraint $x_{s}=\sum_{i=1}^{n_{s}} x_{s, i}\left(P_{s}\right)$ is an upper bound constraint on a convex function in $\left(x_{s}, P_{s}\right)$, problem (16) can be turned into maximizing a strictly concave objective function over a convex constraint set. Therefore, the established convergence is towards the global optimal.

\section{Extension: Some Practical Issues for Application}

\subsection{Fairness}

Packets originating at vehicles outside the communication range of gateway must undergo several channel contentions to reach the gateway. Hence, they have disadvantages over the packets that travel shorter distance to the gateway. In addition, as stated previously, in the cooperation system, each vehicle may transmit both its own bits as well as some information for its partner, how to keep relative fairness between its own bits and partner's bits is also a practical problem need to be solved.

In generic wireless network, the fairness strategy adopts the per-flow fairness, in which every flow has the equal "opportunity" to get the destination; however, this is impractical in VANET system. Here we can employ a content-based fairness strategy as described in [13], and it is scalable and does not require maintenance of any state information beyond a time slot which is important in a network with a fast changing topology. As to the content-based fairness strategy, that is when each node receives 
the packets, it judges the packet content whether it has hold or not. If has, it will throw out the packet directly and inform his partner do not transmit after that moment. If not, it will receive the packet and adjust the flow rate and persistence probability as stated before.

In addition, utility functions can also be interpreted as the "knobs" to control the tradeoff between the fairness and efficiency. Different shapes of utility functions lead to different types of fairness defined in the economics literature. For example, a family of utility functions, parameterized by $a \geq 0$, is proposed in [14]

$$
U^{\alpha}(x)= \begin{cases}(1-\alpha)^{-1} x^{1-\alpha}, & \text { if } \alpha \neq 1 \\ \lg x, & \text { otherwise }\end{cases}
$$

If we set $a=0$, utility maximization reduces to system throughput maximization. If $a=1$, proportional fairness among competing vehicles is attained; if $a=2$, then harmonic mean fairness; and $a \rightarrow \infty$, then max-min fairness. To accommodate multi-class services and attain the desired tradeoff between efficiency and fairness, it is important that the utility maximization framework can handle general types of convex/concave utility functions.

\subsection{Network Cost}

In our system model, we have only considered the user utility. We can introduce a variable $\omega_{l}$ for each link $l$ to represent the cost incurred by using the link to transmit flow to destination. Our objective is to maximize net-gain to strike a balance between vehicle utilities and network cost:

$$
\sum_{s \in S} U_{s}\left(x_{s}\right)-\sum_{l \in L} \omega_{l} c_{l}
$$


Link cost $\omega_{l}$ can be a function of link state such as loss rate, or any other link metric. Following the analysis described in Section 3, we can obtain similar cross layer MAC, rate control and routing algorithm as followings. Change the equation (19) into

$$
x_{s, i}(t+1)=\left[x_{s, i}(t)-\gamma\left(p_{s}^{r^{*}}(t)-p_{s, i}^{r}(t)-\omega_{s, i}(t)\right)\right]^{+}, \quad \gamma>0 .
$$

All the other steps in JOC algorithm remain the same.

The introduction of $\omega_{l}$ facilitates the implementation of many functionalities. For example, if it is an increasing function of link loss rate, we can do link-state-aware scheduling and avoid less reliable links. It can also help to improve performance in delay. In our original design, the flows find their way to destinations by moving in directions of increasing persistence probability. Thus, some data may take a long path to its destination, which could lead to significant delay for large network. By taking $\omega$ proportional to the link length, we can align the nodes to route data in the direction of their destinations, and thus improve the performance in delay.

\section{Simulation Results and Discussion}

We conduct simulation experiments to study the performance of the proposed OC-MAC protocol and JOC scheme. First of all, we describe the simulation settings. Secondly, we present the main simulation results where we show the objective performance results under different scenarios comparing to the competing methods. Finally, we conclude this section based on the selected simulation results. 


\subsection{Simulation Settings}

We implement the proposed scheme in Nab network simulator [15], which is a fast, flexible and scalable simulator for ad-hoc networks. We incorporate our mobility model, and traffic model into the simulator. The vehicle (node) arrival process at the access point follows a Poisson distribution with the average inter arrival time varying from 0.5 to 4 seconds. We consider only one direction of vehicle motion in the highway. The neighbor group is maintained among vehicles driving in the same direction. For simulation convenience, there are 5 original source vehicles distributed uniformly in the system, and all of the other vehicles want to download the data from the sources. We use slow fading Rayleigh channel with unit mean to simulate the wireless radio channel. The parameters used in the simulation are listed in Table 1 . We modify the frame structure based on IEEE 802.11, and the new RTS frame is defined as Figure 5, in which the HA field represents the address of the relay node. When a node receives a RTS frame, it will compare this address to its own. If they are the same, it will send out a RC frame, which is defined as Figure 6.

Table 1: Parameter Settings

\begin{tabular}{|c|c|}
\hline MAC header & 272 bits \\
\hline PHY header & 192 bits \\
\hline RTS & 208 bits \\
\hline CTS & 304 bits \\
\hline ACK & 304 bits \\
\hline RS & 160 bits \\
\hline Packet Size & 1024 bits \\
\hline Data rate for MAC and PHY header & 1 M bps \\
\hline Slot time & 20 us \\
\hline SIFS & $10 \mathrm{us}$ \\
\hline
\end{tabular}




\begin{tabular}{|c|c|}
\hline DIFS & 50 us \\
\hline Total number of the nodes & $10-35$ \\
\hline Vehicle velocity & $40-80 \mathrm{mph}$ \\
\hline
\end{tabular}

It should be noted that all the simulation results in this section have been obtained using 300 runs in order to obtain statistically meaningful average values.

\subsection{Selected Simulation Results and Discussions}

At first, to demonstrate the effectiveness of our proposed OC-MAC strategy, we plot the throughput variation as the number of the nodes increases. The proposed OC-MAC is benchmarked against other three methods: 1) conventional client-server scheme (it uses IEEE 802.11 at the MAC layer, and the gateway is the only source). 2) no-relay cooperation approach (it doest not use relay all the time). 3) always-relay cooperation approach (it uses relay all the time, if any). For notational convenience, we note the above three competing schemes as conventional, no-relay and always-relay, respectively. Figure 7 shows the performance comparison between our proposed OC-MAC and the other three competing schemes. The proposed OC-MAC method can be seen to achieve a higher performance in terms of throughput compared to the conventional client-server method. Obviously, this is due to the introduction of the cooperation. For the cooperative system, there are many possible sources to provide the information other nodes may be interested in. It should also be noted from this figure that the performance achieved by the proposed OC-MAC method is also super to the no-relay and always-relay cooperation method. Though proposed 
OC-MAC method achieves almost the same performance compared to the always-relay method when node number is small and the no-relay method when node number is large, however, on the whole, the proposed OC-MAC method always outperforms the above no-relay and always-relay methods. For example, when the node number is 10 , the gaps between the proposed OC-MAC method with the no-relay method and always-relay method are 1.2 and 0 PPS respectively, while the node number is 30 , the gaps are 0.1 and 0.5 PPS, respectively. We also observe that throughput decreases when the number of nodes in the network becomes larger, which is because the more nodes in the network, the higher possibility of the packets collision during a transmission. In addition, with more nodes in the network, the routing protocol needs more routing information exchange to maintain the routing table, so the traffic load increases and leads to the congestion of network. This is the main reason why we provide the joint control algorithm for this cooperative system.

Figure 8 shows the influence of the velocity of nodes on the throughput performance. With the increase of speed, the link quality deteriorates because the reception power decreases. Our proposed OC-MAC can achieve a satisfactory performance because relay nodes keep a copy of the data frame which increases the possibility of correct reception if it is necessary to employ the relay. Generally speaking, a relay node is used when the velocity is large and channel condition is not good. This rule can be easily concluded from this figure. In addition, we note that there is no distinct difference influence between the OC-MAC, the no-relay and always-relay when the velocity is more than $70 \mathrm{mph}$, which is because both the relay and direction channels are not good for transmission in this case. 
And then, we present some simulation results for JOC algorithm. The network with end-to-end paths and logic links is shown in Figure 9. Each of the three sources has its utility function parameterized by $a$ as in (25). We assume that if the distance between the transmitter node and the receiver node is less than $2 \mathrm{~d}$ (see Figure 9), the receiver node gets interfered from the transmitter node. For simplicity, we assume that all of the links capacities are identically and uniformly distributed over one unit.

Figure 10 shows the convergence of link persistence probabilities regulated by JOC algorithm for the case of $a=3$ with step-size $\kappa=\gamma=0.1$ (Note: the initial persistence probability of each link is random distributed from 0 to $1 ; \mathrm{A}, \mathrm{E}$ and $\mathrm{G}$ are source nodes, while the $\mathrm{C}, \mathrm{D}$ and $\mathrm{F}$ are destination nodes). Figure 11 shows the optimized source rates with each data point being the result of solving (16) for a given fairness parameter $a$. A number of interesting observations can be made from this graph. For example, since source 1 traverses more heavily interfered links, at the optimal rate allocation that maximizes the network utility, it is allocated the lowest data rate. However, as the value of $a$ increases, the gap among the source decreases, improving fairness among sources.

Finally, to demonstrate the effectiveness of our proposed OC-MAC protocol combined with JOC algorithm for the cooperative VANET system, we plot the throughput curves at the node velocity equal to $40 \mathrm{mph}$ and $80 \mathrm{mph}$, respectively. The proposed OC-MAC+JOC scheme is benchmarked against the scheme with only OC-MAC, in which it does not employ any control algorithm. The performance comparison is illustrated in Figure 12. The proposed OC-MAC+JOC method can be 
seen to achieve a higher performance in terms of throughput. When the velocity of the node is $40 \mathrm{mph}$ and the number of the node ranges from 10 to 35 , the average throughput using the proposed scheme is about 8.73 PPS while it is 8.12 PPS for the case of OC-MAC method, thus, around 0.6 PPS performance gain can be achieved on average using the proposed OC-MAC+JOC scheme. Similarly, when the velocity of the node is $80 \mathrm{mph}$, around 0.5 PPS performance gain can be achieved on the average.

\subsection{Observations}

Based on the selected objective and subjective simulation results described above, we conclude the following three main observations:

- Opportunistic cooperation strategy (OC-MAC protocol) can improve the throughput of the VANET system compared to the conventional IEEE 802.11 standard client-service system. In addition, it also outperforms the no-relay and always-relay approaches in terms of adaptability (please see Figure 7 and Figure 8).

- Joint optimal control algorithm actually can adjust flow rates at Transport layer according to the link persistence probability adjusted at the MAC layer. The simulation results support the previous theory analysis that the proposed JOC algorithm converges to the globally optimal solutions (please see Figure 10 and Figure 11).

- From the objective comparisons, it is clear that the proposed OC-MAC combined with JOC scheme outperforms the only OC-MAC method and can provide a satisfactory service for practical VANET system (please see Figure 
12).

\section{Related Works}

For the single-source-single-path utility maximization problem, i.e., each vehicle has only one available path, had been extensively studied in the past, mainly in the context of Internet rate control (for example, [16-17] and the reference therein). In addition, single-source-multi-path utility maximization problem also had received much attention in many literatures (for example, [18], [7] and the reference therein). [18] categorizes the solutions into primal algorithms and dual algorithms. Globe convergence of the primal algorithms is studied in [18] for the case of when feedback delays are negligible and the oscillation problem of dual algorithm are discussed in [6]. In [7], the authors propose two flow control algorithms for network with multiple paths between source-destination pair, both are distributed algorithms over the network to maximize aggregate source utility. In contrast to the abundance of methods proposed to deal with single source utility problem, little attention has been received on multi-source-multi-path problem. [7] studies utility maximization problem for communication networks where each vehicle can have multiple alternative paths and develops a distributed solution to this problem that was amenable to online implementation.

The work in [18], [19], [16], [12] provides a utility-based optimization framework for Internet congestion control. The same framework has been applied to study the congestion control over ad hoc wireless networks (see, e.g., [20], [21]). [21] studies 
joint congestion control and media access control for ad hoc wireless network, and formulates rate allocation as a utility maximization problem with the constraints that arise from contention for channel access. [22] uses a similar model to study this problem by jointly routing the flows and scheduling the transmissions to determine the achievable rates in multi-hop wireless networks. To sum up, all these works focus on the interaction between link and network layers, and try to characterize the achievable rate region at network layer.

Cross-layer design in communication networks, especially in wireless networks, has attracted great attention recently (see, e.g. [23] for an overview). Our work belongs to the category of cross-layer design via dual decomposition in optimization framework. Other work that can be put into this category includes TCP/IP interaction in [24], joint routing and resource allocation in [11] and joint TCP and power control in [10]. The work on joint rate control, MAC and routing design is the first step to provide a unified framework for systematically carrying out cross-layer design. We will extend the framework to include other layers in the future.

\section{Concluding Remarks}

In this paper, we have been focusing on optimizing the rate control, medium access control and routing problem jointly for cooperative VANET. Firstly, we introduce the cooperative communication conception to VANET, and propose an opportunistic cooperation strategy to improve the system throughput. Secondly, we develop a cross-layer control algorithm by joint working at medium access control layer to 
adjust link persistence probability, at the transport layer to adjust flow rate and at the network layer to determine the routing. Furthermore, some practical issues for implementing the proposed algorithm and improving the system performance are presented. The simulation results show that the effectiveness of the proposed opportunistic cooperation strategy combined with joint control algorithm for the cooperative VANET.

\section{Acknowledgments}

This work is supported by the International Project PRA-SI (financed by France and China government) under Grant No.SI04-03, and the Key Project of Nature Science Foundation of Jiangsu (China) under Grant BK2007729. What's more, we also thank the anonymous reviewers for insightful comments and suggestions.

\section{Appendix: Proof of Strictly Concave Function}

Proof: Taking derivatives again, for each of the nonlinear
$\sum_{i=1}^{n_{s}} \log \left[c_{l} p_{l} \prod_{k \in N_{\text {to }}^{I}(l)}\left(1-\sum_{m \in L_{\text {out }}(k)} p_{m}\right)-\sum_{n \in L_{\text {from }}^{I}} c_{n} p_{n} \prod_{k \in N_{\text {to }}^{\prime}(n), k \neq t_{l}}\left(1-\sum_{m \in L_{\text {out }}(k)} p_{m}\right)\right]$ terms in $L_{P}(\tilde{P})$, we obtain the Hessian

$$
H^{l}=\frac{-p_{l}}{\left(\sum_{k} z_{l k}+c_{l} p_{l}\right)^{2}}\left(\left(\sum_{k} z_{l k}+c_{l} p_{l}\right) \operatorname{diag}\left(z_{l}\right)-z_{l} z_{l}^{T}\right)
$$

where $z_{l k}=\exp \left(\tilde{p}_{l}\right)+c_{l} p_{l} \prod_{k \in N_{l o}^{I}(l)}\left(1-\sum_{m \in L_{\text {out }}(k)} p_{m}\right)$, and $z_{l}$ is a column vector $\left[z_{l 1}, z_{l 2}, \ldots z_{l l}\right]^{T}$.

Matrix $H^{l}$ is indeed negative definite: for all vectors $v$ 


$$
v^{T} H^{l} v=\frac{-p_{l}\left[\left(\sum_{k} z_{l k}+c_{l} p_{l}\right)\left(\sum_{k \in N_{l o}^{l}(l)}\left(1-\sum_{m \in L_{\text {out }}(k)} p_{m}\right) z_{l k}\right)-\left(\sum_{k \in N_{l o}^{I}(l)}\left(1-\sum_{m \in L_{\text {out }}(k)} p_{m}\right) z_{l k}\right)^{2}\right]}{\left(\sum_{k} z_{l k}+c_{l} p_{l}\right)^{2}}<0
$$

This is because the Cauchy Schwarz inequality $\left(a^{T} a\right)\left(b^{T} b\right) \geq\left(a^{T} b\right)^{2}$. Therefore, $L_{P}(\tilde{P})$ is a strictly concave function of $\tilde{P}$, and its Hessian is a negative definite block diagonal matrix $\operatorname{diag}\left(H^{1}, H^{2}, \ldots . H^{l}\right)$.

\section{Reference}

[1] A. Nosratinia, T. E. Hunter, A. Hedayat, "Cooperative communication in Wireless Networks”, IEEE Communication Magazine, vol. 42, no.10, pp.74-80, Oct. 2004

[2] Nandan, A., Das, S., Pau, G., etc., "Co-operative downloading in vehicular ad-hoc wireless networks", WONS 2005, pp.32 -41.

[3] Yuan, J., Li, Z., Yu, W., Li, B, "A Cross-Layer Optimization Framework for Multihop Multicast in Wireless Mesh Networks", IEEE Journal on Selected Areas in Communications, vol.24, no.11, pp. 2092 - 2103, Nov. 2006

[4] www.astm.org/SNEWS/MAY 2004/dsrc may04.html

[5] Pei Liu, Zhifeng Tao, Sathya Narayanan, etc. "CoopMAC: A Cooperative MAC for Wireless LANs", IEEE Journal on Selected Areas in Communications, vol.25, no.2, February, 2007, pp.340 - 354.

[6] X. Lin, Ness B. Shroff, "Utility Maximization for Communication Networks with Multi-path Routing”, IEEE Trans. on Automatic Control, vol.51, no.5, pp.766-781, May 2006.

[7] W.H. Wang, M. Palaniswami, and S. H. Low, "Optimal Flow Control and Routing in Multi-Path Networks", Performance Evaluation, vol. 52, no. 2-3, pp. 119-132, April 2003.

[8] L. Chen, Steven H. Low, Mung Chiang, and John C.Doyle, "Cross-layer Congestion Control, Routing and Scheduling Design in Ad Hoc Wireless Networks", INFOCOM 2006. 
[9] R. T. Rockafellar, "Monotone Operators and the Proximal Point Algorithm", SIAM J. Control and Optimization, vol. 14, pp. 877-898, Aug. 1976.

[10] M. Chiang, "To layer or not to layer: balancing transport and physical layers in wirless multihop networks", Proc. IEEE INFOCOM, vol.4, pp.2525-2536, Mar. 2004.

[11] L. Xiao. M. Johnasson and S. Boyd, "Simultaneous routing and resource allocation via dual decomposition”, IEEE Trans. on Communications, vol.52, no.7, pp. 1136-1144, July 2004.

[12] S. H. Low, "A duality model of TCP and queue management algorithms", IEEE/ACM Trans. on Networking, vol. 11, no.4, pp. 525-536, Aug. 2003.

[13] J. Mo and J. Walrand, "Fair end-to-end window-based congestion control," IEEE/ACM Trans. on Networking, vol. 8, no. 5, pp. 556-567, October 2000.

[14] J.-W. Lee, M. Chiang, and A. R. Calderbank, "Jointly optimal congestion and medium access control based on network utility maximization", IEEE Communications Letters, Vol. 10, No. 3, Mar. 2006, pp. 216-218.

[15] Henri-Dubios Ferriere, http://nab.epfh.ch

[16] S. H. Low and D. E. Lapsley, "Optimization flow control-I: basic algorithm and convergence”, IEEE/ACM Trans. on Networking, vol. 7, no. 6, pp. 861-874, Dec. 1999.

[17] H. Yaiche, R.R. Mazumdar, and C. Rosenberg, "A Game Theoretic Framework for Bandwidth Allocation and Pricing in Broadband Networks", IEEE/ACM Trans. on Networking, vol. 8, no. 5, pp. 667-678, Oct. 2000.

[18] F. P. Kelly, A. Maulloo, and D. Tan, "Rate Control in Communication Networks: Shadow Prices, Proportional Fairness and Stability", Journal of the Operational Research Society, vol. 49, no. 3, pp. 237-252, Mar. 1998.

[19] S. Kunniyur and R. Srikant, "End-to-end congestion control schemes: Utility functions, random losses and ECN marks", IEEE/ACM Transactions on networking, 11(5):689-702, October 2003.

[20] Y. Yi and S. Shakkottai, "Hop-by-hop congestion control over a wireless multi-hop network", Proc. IEEE Infocom, vol. 4, Mar. 2004, pp.2548-2558.

[21] L. Chen, S. H. Low and J. C. Doyle, "Joint congestion control and media access control design for ad hoc wireless networks", Proc. IEEE Infocom, vol. 3, Mar.2005, pp. 2212-2222. 
[22] M. Kodialam and T. Nandagopal, "Characterizing achievable rates in multi-hop wireless networks: the joint routing and scheduling problem", Proc. ACM Mobicom, September 2003, pp.42-54.

[23] S. Shakkottai, T. S. Rappaport and P. C. Karlsson, "Cross layer design for wireless networks", IEEE Communications Magazine, vol.41, no.10, Oct. 2003, pp. 74-80.

[24] J. Wang. L. Li, S . H. Low and 3. C. Doyle, "Can TCP and shortest-path routing maximize utility?” Proc, IEEE Infocom. Vol.3, April 2003, pp.2049-2056. 